This is the final peer-reviewed accepted manuscript of:

The ATP synthase glycine zipper of the $c$ subunits: from the structural to the functional role in mitochondrial biology of cardiovascular diseases.

Nesci S, Rubattu S. Biochim Biophys Acta Mol Cell Res. 2021, 1868(9):119075.

The final published version is available online at: https://doi.org/10.1016/j.bbamcr.2021.119075

Rights / License:

The terms and conditions for the reuse of this version of the manuscript are specified in the publishing policy. For all terms of use and more information see the publisher's website.

This item was downloaded from IRIS Università di Bologna (https://cris.unibo.it/)

When citing, please refer to the published version. 


\section{The ATP synthase glycine zipper of the $c$ subunits: from the structural to the functional role in mitochondrial biology of cardiovascular diseases.}

The holo-ATP synthase has a membrane rotor engine embedded in the inner mitochondrial membrane (IMM) formed by the $c$-ring structure in the $F_{0}$ hydrophobic domain [1]. Packing several copies of the $c$ subunits provides a palisade of hairpin-shaped $\alpha$-helices. The stoichiometry of the $c$-ring is specie-dependent and leads to a different $c$-ring size. Each hydrophobic hairpin shaped $c$ subunit constitutes the basic unit of the ring: the $\mathrm{N}$ - and $\mathrm{C}$-terminal $\alpha$-helices of each subunit flank the same terminal regions of another subunit. This assembly forms two concentric circles with the $\mathrm{N}$ - and $\mathrm{C}$ - terminal $\alpha$-helices that form the tight and wide transmembrane $\alpha$-helix packing of the $c$-ring, respectively (Fig. 1A). In eukaryotes, these two circles in each $c$ subunit are linked by a loop region which faces the headgroup region of phospholipids on the matrix side of the IMM and forms the contact site of the feat of the central stalk ( $\gamma, \delta$, and $\varepsilon$ subunits). The hole of the $c-$ ring is occupied with phospholipids [2]. In detail, the recent cryo-EM structure of the entire mammalian ATP synthase [3] reports lipids into the central hole from both sides of the cylinder. In the $c$-ring at the positive side of IMM a lipid plug, probably a lyso-phosphatidylserine, is linked by the terminal lysine of $e$ subunit, whereas at the matrix side the $\mathrm{Arg}^{38}$ of the $c$ subunit on the $\mathrm{N}$-terminal $\alpha$-helix coordinates electrostatically the phosphatidylserine. The two phospholipids are separated in the middle of the $c$-ring by $\mathrm{Val}^{16}$ of $c$ subunits (Fig. 1B). The single acyl-chain of lyso-phosphatidylserine inside the $c$-ring hole has enough space around it. Conversely, the double acyl-chains of phosphatidylserine are a tight fit to the $c$ subunits. This strongly suggests + sit the lyso-phosphatidylserine does not rotate with the $c$-ring, acting as a "lubricated" lipid plug on t! positive side of iMivil, while the matrix-side phesphatidylserine does [3]. It has been established that the glycine zipper domain of the $c$ subunit (Fig. 1.B) Sundles the lipid acyl-chains into the cavity of the rotor, apart from playing a specific iirluence on the $c$-ring stoichiometry that fulfils the mechanism of biological

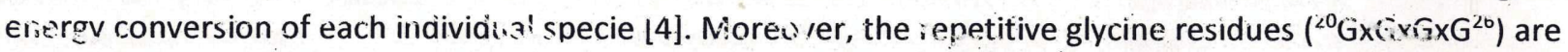
i: ring stability [5]. The degree of structural compliance in the assembly of the rotor and geometry relies on $i$ ) the hydrophobic and weak $\mathrm{H}$-bonds mix interactions arise from glycine zipper and ii) limited steric hindrance of Gly side chain (hydrngen atom). Probably, for this feature, the ${ }^{20} \mathrm{GxGxGxG}{ }^{26}$ motif can play a central role in the permeability transition pore complex (PTPC) opening [6].

The activation oi ATP synthase by an abrupt increase of $\mathrm{Ca}^{2+}$ in mitochondria can trigger the PTPC phenomenon [7]. The new insights on the ATP synthase functions reveal at the molecular level the biological feature of the enzyme complex PTPC formation [8] as hypothesized by the lipid-plug out $c$-ring release in the "bent-pull" model [9]. Accordingly, the PTPC opens when the ATP synthase assumes the disassembled conformation in the presence of $\mathrm{Ca}^{2+}$ bound to the catalytic sites of $F_{1}$, the hydrophilic domain of the enzyme. In this state, the peripheral stalk that joins the $F_{1}$ to $F_{0}$ is twisted with a consistent structural difference compared to the conformation in the presence of the natural cofactor $\mathrm{Mg}^{2+}$. The structural change is transduced in signal propagation along with the entire enzyme from the catalytic site to the $e$ subunit. Lastly, the lyso-phosphatidylserine is expelled from the central hole inside the $c$-ring and opens the channel at the positive side of IMM. The other side of the $c$-ring is filled with phosphatidylserine that can block ion conductance until the lipid is pushed apart by the hole. Simultaneously, water molecules fill the $c$-ring destabilizing the phosphatidylserine on the matrix side and the $F_{1}$ detaches from $F_{0}$. The final result is the ejection of the phosphatidylserine from the central hole of the $c$-ring and the PTPC opening [3].

In physiological conditions, the opening of PTPC is involved in the cardiac and neural development. The relevance of a modulation of PTPC activity in this context is supported by evidence that depletion of the main activator of the PTPC, cyclophilin D, causes cardiac myocyte differentiation to begin earlier [9]. 
In pathological conditions, it has been well assessed that PTPC opening behaves as a key driver during myocardial ischemia/reperfusion ( $(/ / R)$ injury [10]. During ischemia cardiomyocytes are exposed to $\mathrm{Ca}^{2+}$ overload, increased oxidative stress and inflammation. These factors are known to favour the susceptibility of the PTPC to opening. In addition, once reperfusion occurs, it restores a normal $\mathrm{pH}$ which induces increased PTPC activity with consequent severe mitochondrial impairment and disruption of energy production, leaúing to a massive cellular damage [11]. Accordingly, acidic $\mathrm{pH}$ reduces cell death in $\mathrm{I} / \mathrm{R}$, and this has been attributed to inhibition of the PTPC opening by acid $\mathrm{pH}$. Of importance, there is evidence that inhibitors of the PTPC, i.e. cyclosporin A, may reduce infarct size following $I / R$ in humans [12] although a robust demonstration of this phenomenon is still lacking.

The link between inhibition of the PTPC opening and cardioprotection has been demonstrated in cells, isolated hearts, and in-vivo models [10]. Apart from the $\mathrm{I} / \mathrm{R}$ and the myocardial infarct conditions, a role of PTPC opening has been hypothesized, but not yet definitively demonstrated, in heart failure [10].

Recent data gained on the glycine-rich region of $c$ subunit provide useful information for a better understanding of the molecular mechanism that leads to the PTPC phenomenon and of its involvement in cardiovascular diseases. Indeed, glycine zipper of $c$ subunits can be a critical factor in exacerbating PTPC opening under pathological conditions [13]. Gly residue is the only amino acid without a side chain. The substitution of Gly with other amino acids necessarily produces a steric hindrance and much more interactions than the $\mathrm{H}$ atom alone. Increased channel conductance of mutated glycine zipper suggests decreased packing anủ a iarge central pore formation [14]. Different substitution of Gly ${ }^{26}$ would exhibit a structural deformation at the level of the $\mathrm{N}$-terminai helix that differently modulates the PTPC opening [6]. Moreover, Gly ${ }^{26}$ mutation is more sensitive to form PTPC than mutation on Gly ${ }^{24}$. Gly $y^{22}$. and Gly ${ }^{20}$ and more tr-an wild ofe $c$ subunit. In particular, the Gly ${ }^{26} \mathrm{Glu}$ mis ation within the gly cone-rich region of $c$ subunit causes the alteration $c^{\prime}$ the side chain length and the addition of negatively criargea residue that could favour the instability of phosphatidylserine in tie $c$-ring. Therefire when the ATP synthase triggers the PTPC forriation, the mutant $r$ sunit may wiefer a hydrophilic hole int wing for the pursage of water.

In this context, the pathological role of the naturally occurring mutation ATP5G ${ }^{687 E}$ within the $c$ subunit has been recently unraveled by the elegant work of Morciano et al. [13]. The authors of the study showed that carriers of this mutation suffered from a greater myocardial damage at the time of reperfusion following STelevation wiyocardial farction (STEMI). A significant inverse correlation was detected between PTPC activity and reperfusion damage in this condition, in particular, Gly ${ }^{26} \mathrm{Glu}$ substitution exhibited hyperresponsive PTPC opening compared with wild type $c$ subunit by aggravating the PTPC-mediated hypoxia/reoxygenation cell death in human cardiomyocytes [13]. These effects are consistent with the abovementioned notion that PTPC remains closed during myocardial ischemia and re-opens at the time of reperfusion. The human evidence is corroborated by the consequences of genetic manipulations of $c$ subunit expression levels by siRNA in HeLa cells. Herein, the in-vitro data confirmed that $c$ subunit is required for PTPC driven mitochondrial fragmentation and cell death triggered by cytosolic $\mathrm{Ca}^{2+}$ overload and oxidative stress $[14,15]$.

The evidence so far gained on the pathogenic implications of $c$ subunit in ischemic heart disease raises another interesting issue, that is its potential therapeutic implications to improve treatment of this common disease and to reduce its dramatic consequences. In fact, several efforts have been already made in the attempt to reduce the tissue damage at the time of reperfusion, but none turned out successful. The discovery that the ATP5G $1^{\mathrm{G} 87 \mathrm{E}}$ mutation, within the highly conserved glycine-rich domain of the $c$ subunit, amplifies the reperfusion injury by further enhancing PTPC opening reinforces the hypothesis that a timely intervention with inhibitors of PTPC activity may serve as a valid therapeutic approach to reduce the hypoxia/reoxygenation damage at the time of revascularization of an occluded coronary artery. In particular, the strategy based on the selective targeting of $c$ subunit, to reduce PTPC opening, may reveal the most suitable approach during coronary angioplasty following myocardial infarction. Moreover, by reducing the 
myocardial damage dependent from coronary occlusion and particularly from the subsequent revascularization, a decrease of the cardiac remodelling process and a reduced progression of ischemic cardiomyopathy toward heart failure development would be expected.

The emerging link of the ATP synthase $c$ subunit with cardiovascular diseases deserves to be fully dissected out through future experimental and human studies.

Salvatore Nesci ${ }^{1}$, Speranza Rubattu ${ }^{2,3}$

${ }^{1}$ Department of Veterinary Medical Sciences, Alma Mater Studiorum University of Bologna, 40064 Ozzano Emilia, Italy. ${ }^{2}$ Department of Clinical and Molecular Medicine, School of Medicine and Psychology, "Sapienza" University of Rome, Rome 00189 , Italy. ${ }^{3}$ RCCS Neuromed, Pozzilli, IS 86077 , Italy.

Corresponding authors: salvatore.nesci@unibo.it (S. Nesci): rubattu.speranza@neuromed.it (S. Rubattu)

\section{References}

[1] S. Nes.i, A. Pagliarani C. Agieri, F. Trombetti, Mitorhondrial F-type ATP synthase: multiple enz;me functions revealed by the meiibrane-embedded Fo structure, Crit. Fiev. Biochem. Mo Biol. 55 (2020) 309-21. htips///doi.org/10.1080/10409238.2020 1784084.

[2] B. Oberteld, I Brunne, $D$ D.moth Phuspholipids occupy the internat wmen of the ring of the AfF

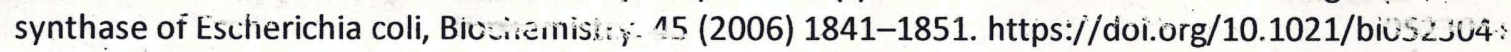

[3] G. Pinke, L. Zhou, L.A. Sazanov, Cryo-EM structure of the entire mammalian F-type ATP synthase, Nat Struct Mol Biol. 27 (2020) 1077-1085. https://doi.org/10.1038/s41594-020-0503-8.

[4] D. Pogoryelov, A.L. Klyszejko, G.O. Krasnoselska, E.-M. HeHer, V. Leone, J.D. Lànger, J. Vonck, D.J. Müller, J.D. Faraldo-Gótroz, T.Mciar, Engineering rotor ring stoichiometries in the ATP synthase, Proc. Natl. Acad. Sci. U.S.A. 109 (2012) E1599 1608 https://doi.org/10.1073/pnas.1120027109.

[5] L. Preiss, A.L. Klyszejko, D.B. Hicks, J. Liu, O.J.' Fackelmayer, Ö. Yildiz, T.A. Krulwich, T. Meier, The c-ring stoichiometry of ATP synthase is adapted to cell physiological requirements of alkaliphilic Bacillus pseudofirmus OF4, Proc Natl Acad Sci U S A. 110 (2013) 7874-7879.

https://doi.org/10.1073/pnas.1303333110.

[6] M. Bonora, C. Morganti, G. Morciano, G. Pedriali, M. Lebiedzinska-Arciszewska, G. Aquila, C. Giorgi, P. Rizzo, G. Campo, R. Ferrari, G. Kroemer, M.R. Wieckowski, L. Galluzzi, P. Pinton, Mitochondrial permeability transition involves dissociation of F1FO ATP-synthase dimers and C-ring conformation, EMBO Rep. 18 (2017) 1077-1089. https://doi.org/10.15252/embr.201643602.

[7] S. Nesci, A. Pagliarani, Ca2+ as cofactor of the mitochondrial $\mathrm{H}^{+}$-translocating $\mathrm{F}_{1} \mathrm{~F}_{\mathrm{O}}-\mathrm{ATP}$ (hydrol)ase, Proteins: Structure, Function, and Bioinformatics. 89 (2021) 477-482. https://doi.org/10.1002/prot.26040.

[8] S. Nesci, A. Pagliarani, Incoming news on the F-type ATPase structure and functions in mammalian mitochondria, BBA Advances. 1 (2021) 100001. https://doi.org/10.1016/j.bbadva.2020.100001.

[9] N. Mnatsakanyan, E.A. Jonas, ATP synthase c-subunit ring as the channel of mitochondrial permeability transition: Regulator of metabolism in development and degeneration, J. Mol. Cell. Cardiol. 144 (2020) 109-118. https://doi.org/10.1016/j.yjmcc.2020.05.013. 
[10] T.M. Bauer, E. Murphy, Role of Mitochondrial Calcium and the Permeability Transition Pore in Regulating Cell Death, Circ Res. 126 (2020) 280-293. https://doi.org/10.1161/CIRCRESAHA.119.316306.

[11] J.Q. Kwong, J.D. Molkentin, Physiological and pathological roles of the mitochondrial permeability transition pore in the heart, Cell Metab. 21 (2015) 206-214. https://doi.org/10.1016/j.cmet.2014.12.001.

[12] C. Piot, P. Croisille, P. Staat, H. Thibault, G. Rioufol, N. Mewton, R. Elbelghiti, T.T. Cung, E. Bonnefoy, D. Angoulvant, C. Macia, F. Raczka, C. Sporiouch, G. Gahide, G. Finet, X. André-Fouët, D. Revel, G. Kirkorian, J.-P. Monassier, G. Derumeaux, M. Ovize, Effect of cyclosporine on reperfusion injury in acute myocardial infarction, N Engl J Med. 359 (2008) 473-481. https://doi.org/10.1056/NEJMoa071142.

[13] G. Morciano, G. Pedriali, M. Bonora, K. Pavasini, E. Mikus, S. Calvi, M. Bovolenta, M. LebiedzinskaArciszewska, M. Pinotti, A. Albertini, M.R. Wieckowski, C. Giorgi, R. Ferrari, L. Galluzzi, G. Campo, P. Pinton, A naturally occurring mutation in ATP synthase subunit $c$ is associated with increased damage following hypoxia/reoxygenation in STEMI patients, Cell Rep. 35 (2021) 108983. https://doi.org/10.1016/j.celrep.2021.108983.

[14] K.N. Alavian, G. Beutner, E. Lazrove, S. Sacchetti, H.-A. Park, P. Licznerski, H. Li, P. Nabili, K. Hockensmith, M. Graham, G.A. Porter, E.A. Jonas, An uncoupling channel within the c-subunit ring of the F1FO ATP synthase is the mitochondrial permeability transition pore, Proc. Natl. Acad. Sci. U.S.A. 111 (2014) 10580-10585. https://doi.org/10.1073/pnas.1401591111.

[15] M. Bonora, A. Bononi, E. De Marchi, C. Giorgi, M. Lebiedzinska, S. Marchi, S. Patergnani, A. Rimessi, J.M. Suski, A. Wojtala, M.R. Wieckowski, G. Kroemer, L. Galiuzzi, P. Pinton, Role of the c subunit of the FO ATP synthase in mitochondrial permeability transition, Cell Cycle. 12 (2013) 674-683. hittps://doi.org/10.161/cc 33599.

\section{rig̣ure caption}

Figure 1. Structure representation of $c$-ring. A) Cryo-EM of $c$-ring ATP synthase, $8 c$ subunits, PDB ID codes: 6TT7. Lipid-plug int the $c$-ring viewed from the positive side of IMM (left panel) and from matrix side (right panel). B) Cross sectiun of the c-ring. Phosphatidylserine (PS), as negatively chargeu lipid, at the matrix side is coordinated by the positive charge of Arg ${ }^{38}$ residue of $c$ subunits. Lyso-phospatidylserine (LPS) at the positive side of ivivin is bound with its polar head to the Lys $^{71}$ of the $e$ subunit (not shown). Hole lipid chambers are separated by the annular of $\mathrm{Val}^{16}$ residues. In the boxes the structure of both wild-type (left panel) and mutant (right panel) glycine zipper are shown. PS and LPS are drawn as stick models, whereas amino acid residues are drawn as ball-and-stick. 
A)

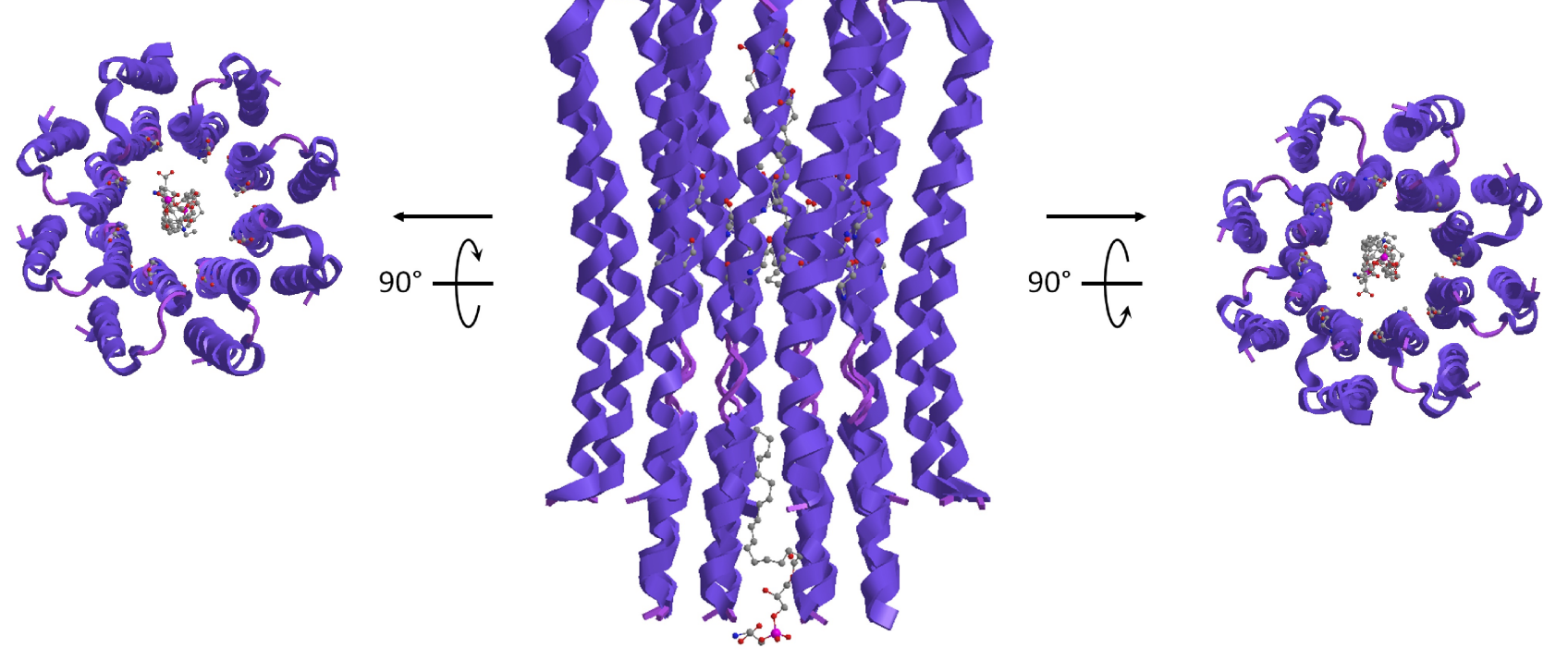

B)
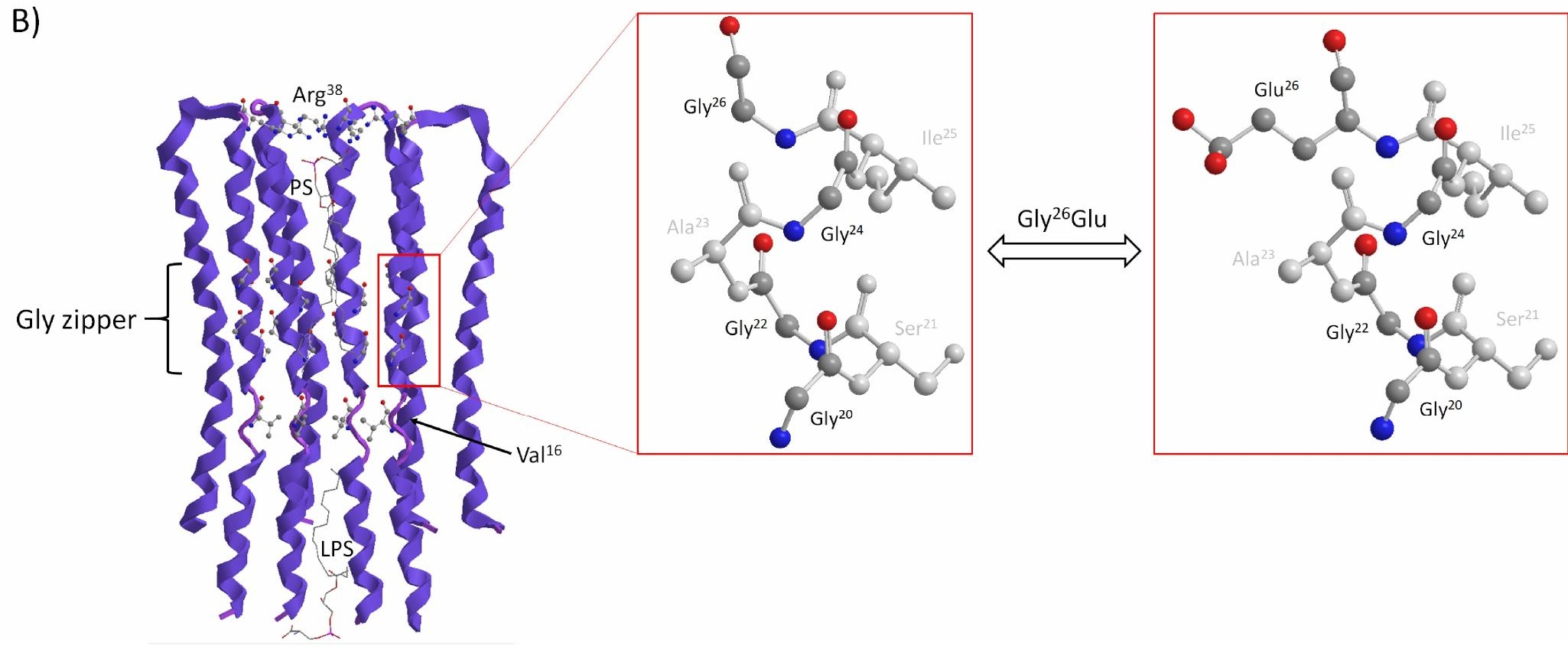